\title{
On combinatorial coefficients and the Gelfond-Khovanskii residue formula
}

\author{
Ivan Soprounov
}

\begin{abstract}
The Gelfond-Khovanskii residue formula computes the sum of the values of any Laurent polynomial over solutions of a system of Laurent polynomial equations whose Newton polytopes have sufficiently general relative position. We discuss two important consequences of this result: an explicit elimination algorithm for such systems and a new formula for the mixed volume. The integer coefficients that appear in the Gelfond-Khovanskii residue formula are geometric invariants that depend only on combinatorics of the polytopes. We explain how to compute them explicitly.
\end{abstract}

\section{Introduction}

A Laurent polynomial is a finite linear combination of monomials with complex coefficients:

$$
f(t)=\sum_{k \in \mathbb{Z}^{n}} \lambda_{k} t^{k}, \quad t^{k}=t_{1}^{k_{1}} \ldots t_{n}^{k_{n}}, \quad k=\left(k_{1}, \ldots, k_{n}\right), \quad \lambda_{k} \in \mathbb{C} .
$$

The convex hull of those lattice points $k \in \mathbb{Z}^{n}$ for which $\lambda_{k} \neq 0$ is a convex polytope in $\mathbb{R}^{n}$, which is called the Newton polytope of $f$. The value of $f(t)$ is defined for all $t$ in the set $(\mathbb{C} \backslash 0)^{n}=\left\{t \in \mathbb{C}^{n} \mid t_{1} \ldots t_{n} \neq 0\right\}$.

In this paper we talk about systems of $n$ Laurent polynomial equations in $(\mathbb{C} \backslash 0)^{n}$ whose Newton polytopes have sufficiently general relative positions (the precise definition will be given in Section 2.1). O. Gelfond and A. Khovanskii [GK02] discovered that one can say a lot about solutions of such systems explicitly in terms of the Newton polytopes and the coefficients of the system. The following theorem ([GK02], Corollary 1.6) illustrates that.

TheOREM 1. Consider a system of $n$ Laurent polynomial equations

$$
f_{1}(t)=\cdots=f_{n}(t)=0, \quad t \in(\mathbb{C} \backslash 0)^{n},
$$

whose Newton polytopes $\Delta_{1}, \ldots, \Delta_{n}$ have sufficiently general relative positions. The sum of the values of a Laurent polynomial $q$ over solutions ${ }^{1}$ of (1) counted with

2000 Mathematics Subject Classification. Primary 14M25; Secondary 52B11.

Key words and phrases. Grothendieck residues, Newton polytopes, combinatorial coefficient. is finite.

${ }^{1}$ The assumption on the position of the polytopes implies that the solution set of the system 
multiplicities is equal to

$$
(-1)^{n} \sum_{A \in \operatorname{vert}(\Delta)} c_{A} \operatorname{res}_{A}\left(q \frac{d f_{1}}{f_{1}} \wedge \cdots \wedge \frac{d f_{n}}{f_{n}}\right)
$$

where the sum is taken over the vertices $A$ of the Minkowski sum $\Delta=\Delta_{1}+\cdots+\Delta_{n}$, $\operatorname{res}_{A}\left(q \frac{d f_{1}}{f_{1}} \wedge \cdots \wedge \frac{d f_{n}}{f_{n}}\right)$ is the residue at the vertex $A$, and $c_{A}$ is the combinatorial coefficient at $A$.

The residue at a vertex $A$ is an explicit Laurent polynomial in the coefficients of $q$ and $f_{1}, \ldots, f_{n}$. In the case when $n=1$ the polytope $\Delta$ is a segment and the residues at the two endpoints of $\Delta$ are the classical residues of $q \frac{d f}{f}$ at zero and infinity. We give the general definition in Section 4.

The coefficients $c_{A}$ are integer numbers which depend only on combinatorics of the polytopes $\Delta_{1}, \ldots, \Delta_{n}$. They can be computed by counting certain complete flags of faces of the Minkowski sum $\Delta$. We discuss this in Section 2.

A particular case of Theorem 1 produces a formula for the mixed volume of the polytopes $\Delta_{1}, \ldots, \Delta_{n}$ in terms of the vertices of the Minkowski sum $\Delta$ and combinatorial coefficients. Furthermore, Theorem 1 provides with an explicit elimination algorithm for systems (1).

The present paper is expository. Proofs of the results that we mention here are contained in [GK02, Kho99, Sop02].

\section{Combinatorial coefficients}

2.1. Definition. Consider a collection of $n$ convex polytopes $\Delta_{1}, \ldots, \Delta_{n}$ in $\mathbb{R}^{n}$. Let $\Delta$ be their Minkowski sum, $\Delta=\Delta_{1}+\cdots+\Delta_{n}$. It is not hard to see that each face $\Gamma$ of $\Delta$ has a unique decomposition into the sum of faces of the $\Delta_{i}$ :

$$
\Gamma=\Gamma_{1}+\cdots+\Gamma_{n}, \quad \Gamma_{i} \text { a face of } \Delta_{i} .
$$

We will call $\Gamma_{i}$ the $i$-th summand of $\Gamma$. A face $\Gamma$ of $\Delta$ is called locked if at least one of its summands has zero dimension, i.e. is a vertex. A vertex $A$ of $\Delta$ is called critical if all the proper faces of $\Delta$ that contain $A$ are locked.

Furthermore, consider a continuous piecewise-linear map $\chi: \Delta \rightarrow \mathbb{R}^{n}$ whose $i$-th component $\chi_{i}$ is non-negative and vanishes precisely on those faces $\Gamma$ of $\Delta$ whose $i$-th summand is a vertex. Such map is called characteristic. Note that the preimage of the origin under a characteristic map $\chi$ consists of the vertices of $\Delta$ only. Also in a neighborhood of each critical vertex $A$ the map $\chi$ sends the boundary $\partial \Delta$ to the boundary $\partial \mathbb{R}_{+}^{n}$ of the positive octant.

Definition 1. The combinatorial coefficient $c_{A}$ at a critical vertex $A$ is the local degree at $A$ of the restriction of $\chi$ to the boundary $\partial \Delta$ :

$$
\chi_{A}:(\partial \Delta, A) \rightarrow\left(\partial \mathbb{R}_{+}^{n}, 0\right) .
$$

One can check that it is independent of the choice of a characteristic map $\chi$ (see [Kho99] for details).

The sign of the combinatorial coefficient depends on the orientation of $\mathbb{R}^{n}$ and the order of the polytopes. Indeed, by definition, the local degree of $\chi_{A}:(\partial \Delta, A) \rightarrow$ $\left(\partial \mathbb{R}_{+}^{n}, 0\right)$ depends on the orientation of the space containing the polytope $\Delta$ and on the orientation of the target space. The latter is determined by the order of the components $\chi_{i}$ of $\chi$, which corresponds to the order of the polytopes. 
Now we will give the precise definition of generic relative position of $n$ polytopes in $\mathbb{R}^{n}$. Let $\Delta_{1}, \ldots, \Delta_{n}$ be convex polytopes in $\mathbb{R}^{n}$. Every linear functional $\xi$ on $\mathbb{R}^{n}$ defines a collection of faces $\Delta_{1}^{\xi}, \ldots, \Delta_{n}^{\xi}$ of the polytopes such that the restriction of $\xi$ to $\Delta_{i}$ attains its minimum precisely at $\Delta_{i}^{\xi}$.

Definition 2. We say that $n$ polytopes $\Delta_{1}, \ldots, \Delta_{n}$ in $\mathbb{R}^{n}$ have generic relative position if for any non-zero linear functional $\xi$ on $\mathbb{R}^{n}$ at least one of the faces $\Delta_{1}^{\xi}, \ldots, \Delta_{n}^{\xi}$ is a vertex.

Note that if $\Delta_{1}, \ldots, \Delta_{n}$ have generic relative position then every proper face of the Minkowski sum $\Delta$ is locked. Therefore the combinatorial coefficient $c_{A}$ is defined at every vertex $A$ of $\Delta$.

2.2. Explicit description. The following theorem gives an explicit description of the combinatorial coefficient as the number of certain complete flags of faces of $\Delta$, counted with signs. The sign of a complete flag

$$
\Gamma^{0} \subset \Gamma^{1} \subset \cdots \subset \Gamma^{n-1} \subset \Gamma^{n}, \quad \operatorname{dim} \Gamma^{i}=i
$$

of faces of a polytope in $\mathbb{R}^{n}$ is the orientation of any frame of vectors $\left(v_{1}, \ldots, v_{n}\right)$, where $v_{i}$ starts at $\Gamma^{0}$ and points strictly inside $\Gamma^{i}$. The sign depends on the orientation of $\mathbb{R}^{n}$.

THEOREM 2. [Sop02] Let $\Delta_{1}, \ldots, \Delta_{n}$ be a collection of convex polytopes in $\mathbb{R}^{n}$ and $\Delta$ their Minkowski sum. The combinatorial coefficient $c_{A}$ at a critical vertex $A$ is equal to the number of all complete flags

$$
A=\Gamma^{0} \subset \Gamma^{1} \subset \cdots \subset \Gamma^{n-1} \subset \Gamma^{n}=\Delta, \quad \operatorname{dim} \Gamma^{i}=i
$$

counted with signs, where $\Gamma^{i}$ is an $i$-dimensional face of $\Delta$ whose first $i$ summands have positive dimensions and the last $n-i$ summands have zero dimension.

Notice that this description is highly non-symmetric in the order of the polytopes. In fact, the actual number of complete flags can be different for different orderings, whereas the signed number of complete flags is always the same (up to a sign), since the combinatorial coefficient is alternating on the order of $\Delta_{1}, \ldots, \Delta_{n}$ as it follows from definition.

2.3. Example. Two plane polygons have generic relative positions if and only if they do not have two parallel sides with the same direction of an inner normal. For example, the square and the triangle on Figure 1 have generic relative position. Each side of the Minkowski sum comes from either one of the polygons. The combinatorial coefficient of a vertex is 0 if both its adjacent sides come from the same polygon, and is \pm 1 otherwise. The sign depends on the orientation defined by the two adjacent sides.

REMARK 1. Combinatorial coefficients first appeared in 1996 in [GK96] where Theorem 1 was announced. The explicit description (Theorem 2) was found in 2001 and provided a connection between the Gelfond-Khovanskii residue formula and Parshin's reciprocity laws (see [Sop02]). It would be interesting to see if this description can be useful in mixed volume computation and elimination process which we discuss in the next section. 

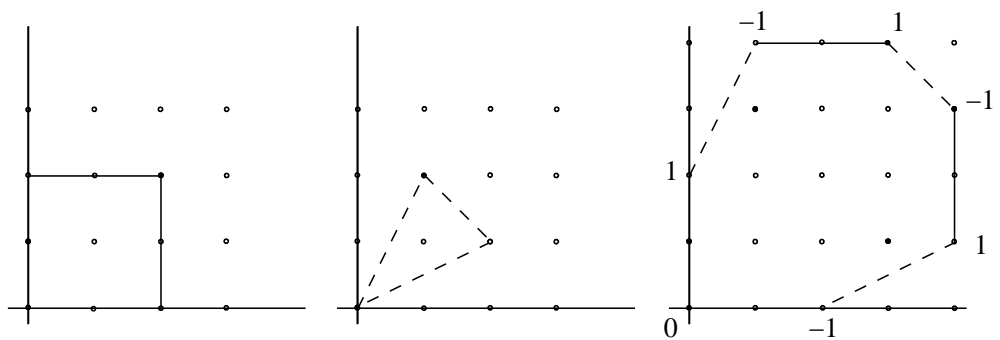

Figure 1. The Minkowski sum of two polygons and combinatorial coefficients.

\section{Applications of Theorem 1}

3.1. Mixed Volume. The special case of Theorem 1 when $q=1$ gives a formula for the number of solutions of the system counting multiplicities. One can prove that the residue $\operatorname{res}_{A}\left(\frac{d f_{1}}{f_{1}} \wedge \cdots \wedge \frac{d f_{n}}{f_{n}}\right)$ is independent of the coefficients of the polynomials $f_{i}$ and equals $\operatorname{det}\left(A_{1}, \ldots, A_{n}\right)$, where $A_{i}$ is the $i$-th summand of the vertex $A$ (see Section 2.1). On the other hand, according to the BKK theorem the number of solutions is $n$ ! times the mixed volume of the Newton polytopes. ${ }^{2}$ This results in a formula for the mixed volume of a collection of $n$ lattice polytopes with generic relative position:

$$
n ! V\left(\Delta_{1}, \ldots, \Delta_{n}\right)=(-1)^{n} \sum_{A \in \operatorname{vert}(\Delta)} c_{A} \operatorname{det}\left(A_{1}, \ldots, A_{n}\right),
$$

where the sum is taken over the vertices $A$ of the Minkowski sum $\Delta, A_{i}$ is the $i$-th summand of $A$, and $c_{A}$ is the combinatorial coefficient at $A$.

Let us remark that this formula holds true for non-lattice polytopes as well. This was proved by Khovanskii [Kho99] using regular polyhedral subdivisions.

3.2. Elimination Algorithm. According to classical elimination theory the projection of the solution set of the system (1) to the $i$-th coordinate line can be given as the solution set of a single polynomial equation in one variable. In fact, Theorem 1 provides us with an explicit algorithm for obtaining such an equation.

For example, let us obtain a polynomial $p\left(t_{1}\right)$ whose roots are the first coordinates of the solutions of the system. First, compute the number $N$ of solutions of the system (counting multiplicities) by taking $q=1$. This is the degree of the polynomial $p$. Then taking $q=t_{1}, t_{1}^{2}, \ldots, t_{1}^{N}$ we get the sums of powers of the roots of $p$. Finally, using the Newton formulas we can express the coefficients of the polynomial $p$ via the sums of powers of its roots. To illustrate this procedure we give an example in Section 5 .

\section{Definition of $\operatorname{res}_{A}\left(q \frac{d f_{1}}{f_{1}} \wedge \cdots \wedge \frac{d f_{n}}{f_{n}}\right)$}

Here we include the definition of the residue $\operatorname{res}_{A}\left(q \frac{d f_{1}}{f_{1}} \wedge \cdots \wedge \frac{d f_{n}}{f_{n}}\right)$ at a vertex $A$ of the Minkowski sum of the Newton polytopes of $f_{1}, \ldots, f_{n}$.

\footnotetext{
${ }^{2}$ The Bernstein-Kushnirenko-Khovanskii theorem says that the number of solutions of a generic system with fixed Newton polytopes is $n$ ! times the mixed volume of the polytopes. However, as it was shown by Khovanskii, if the polytopes have generic relative position then the number of solutions (counting multiplicities) is the same for all systems with given Newton polytopes.
} 
Before we give the general definition let us look at the one-dimensional case. Consider a meromorphic form $q \frac{d f}{f}$, where $q, f$ are Laurent polynomials in one variable. The possible poles of this form are the non-zero roots of $f$, zero, and infinity. The residue of $q \frac{d f}{f}$ at a non-zero root $a$ of $f$ is equal to $\mu(a) q(a)$, where $\mu(a)$ is the multiplicity of the root. Since the total sum of residues is zero the sum of the values of $q$ over the non-zero roots of $f$ counting multiplicities is the negative sum of residues at zero and infinity:

$$
\sum_{a \neq 0, f(a)=0} \mu(a) q(a)=-\left(\operatorname{res}_{0} q \frac{d f}{f}+\operatorname{res}_{\infty} q \frac{d f}{f}\right) .
$$

Recall that $\operatorname{res}_{0} q \frac{d f}{f}$ is the coefficient of $1 / t$ in the Laurent expansion of $q \frac{f^{\prime}}{f}$ at $t=0$ and $-\operatorname{res}_{\infty} q \frac{d f}{f}$ is the coefficient of $1 / t$ in the Laurent expansion of $q \frac{f^{\prime}}{f}$ at $t=\infty$. We denote them by $\operatorname{res}_{A} q \frac{d f}{f}$ and $\operatorname{res}_{B} q \frac{d f}{f}$, respectively, where $[A, B]$ is the Newton polytope (segment) of $f$.

Thus we obtain Theorem 1 for $n=1$ :

$$
\sum_{a \neq 0, f(a)=0} \mu(a) q(a)=-\left(\operatorname{res}_{A} q \frac{d f}{f}-\operatorname{res}_{B} q \frac{d f}{f}\right) .
$$

Note that the combinatorial coefficients are $c_{A}=1$ and $c_{B}=-1$.

The following definition generalizes the notion of the Laurent expansion at zero (infinity) to the case of several variables.

Definition 3. [GK02] Let $f$ be a Laurent polynomial

$$
f(t)=\sum_{k \in \mathbb{Z}^{n}} \lambda_{k} t^{k}
$$

and $A$ a vertex of the Newton polytope of $f$. Then $\lambda_{A} \neq 0$ and so we can write $f=\lambda_{A} t^{A}(1-h)$ for some Laurent polynomial $h$. Consider a series

$$
\frac{1}{f}=\frac{1}{\lambda_{A} t^{A}(1-h)}=\lambda_{A}^{-1} t^{-A}\left(1+h+h^{2}+\ldots\right) .
$$

Note that any monomial can appear only in a finite number of terms $h^{i}$, thus (3) is a well-defined power series. Given a Laurent polynomial $g$, the formal product of $g$ and the series (3) is called the Laurent expansion of $g / f$ at the vertex $A$.

Now we are ready to define the residue $\operatorname{res}_{A}\left(q \frac{d f_{1}}{f_{1}} \wedge \cdots \wedge \frac{d f_{n}}{f_{n}}\right)$. Note that the Newton polytope of the product $f_{1} \ldots f_{n}$ is the Minkowski sum $\Delta$ of the Newton polytopes of $f_{1}, \ldots, f_{n}$. Therefore, the Laurent expansion of $g /\left(f_{1} \ldots f_{n}\right)$ is defined at any vertex $A \in \Delta$ for any Laurent polynomial $g$.

Definition 4. The number $\operatorname{res}_{A}\left(q \frac{d f_{1}}{f_{1}} \wedge \cdots \wedge \frac{d f_{n}}{f_{n}}\right)$ is the coefficient of $\frac{1}{t_{1} \ldots t_{n}}$ in the Laurent expansion of

$$
\frac{q J_{f}}{f_{1} \ldots f_{n}}
$$

at the vertex $A$. Here $J_{f}$ is the Jacobian $\frac{\partial\left(f_{1}, \ldots, f_{n}\right)}{\partial\left(t_{1}, \ldots, t_{n}\right)}$. 


\section{Example}

We now consider an example of a system of two polynomial equations in two unknowns:

$$
f_{1}(x, y)=a_{10} x+a_{01} y+a_{22} x^{2} y^{2}=0, \quad f_{2}(x, y)=b_{00}+b_{12} x y^{2}+b_{21} x^{2} y=0 .
$$

The following are the Newton polygons $\Delta_{1}, \Delta_{2}$ of $f_{1}, f_{2}$ and their Minkowski sum $\Delta$ with the combinatorial coefficients at the vertices.
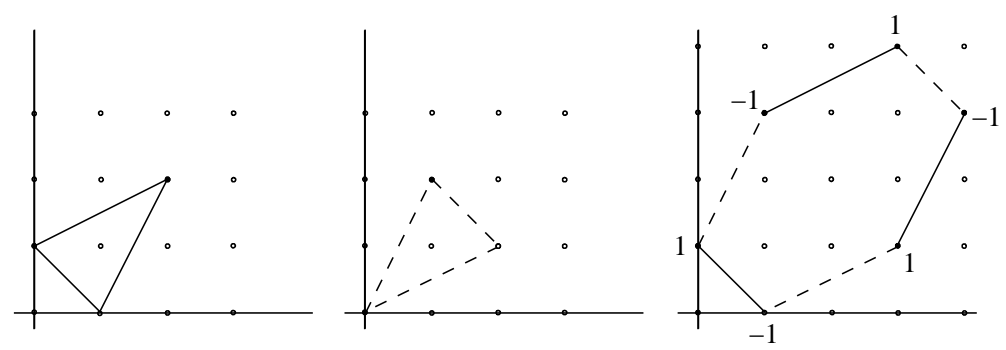

By (2) the number of solutions of the given system (4) (which is twice the mixed volume of $\Delta_{1}, \Delta_{2}$ ) is equal to

$$
N=\left|\begin{array}{ll}
0 & 0 \\
1 & 0
\end{array}\right|-\left|\begin{array}{ll}
0 & 1 \\
1 & 2
\end{array}\right|+\left|\begin{array}{ll}
2 & 1 \\
2 & 2
\end{array}\right|-\left|\begin{array}{ll}
2 & 2 \\
2 & 1
\end{array}\right|+\left|\begin{array}{ll}
1 & 2 \\
0 & 1
\end{array}\right|-\left|\begin{array}{ll}
1 & 0 \\
0 & 0
\end{array}\right|=6 .
$$

We are going to obtain two degree six polynomials in one variable whose roots are the $x$ - and $y$-coordinates of the solution points of (4), respectively.

To make our notations shorter we denote the residue $\operatorname{res}_{A}\left(q \frac{d f_{1}}{f_{1}} \wedge \frac{d f_{2}}{f_{2}}\right)$ at the vertex $A=(l, m)$ by $\operatorname{res}_{(l, m)}(q)$. We compute the residues at the six vertices of $\Delta$ for $q=x^{k}, 1 \leq k \leq 6$ :

$$
\begin{aligned}
& \operatorname{res}_{(0,1)}\left(x^{k}\right)=\operatorname{res}_{(1,0)}\left(x^{k}\right)=\operatorname{res}_{(1,3)}\left(x^{k}\right)=\operatorname{res}_{(3,4)}\left(x^{k}\right)=0, \quad 1 \leq k \leq 6 \\
& \operatorname{res}_{(3,1)}\left(x^{k}\right)= \begin{cases}0, & k=1,2,4,5 \\
\frac{3 b_{00}\left(a_{01} b_{21}-a_{22} b_{00}\right)}{a_{10} b_{21}^{2}}, & k=3 \\
\frac{3 b_{00}^{2}\left(2 a_{10} b_{12}\left(a_{01} b_{21}-2 a_{22} b_{00}\right)+\left(a_{01} b_{21}-a_{22} b_{00}\right)^{2}\right)}{a_{10}^{2} b_{21}^{4}}, & k=6\end{cases} \\
& \operatorname{res}_{(4,3)}\left(x^{k}\right)= \begin{cases}0, & k=1,2,4,5 \\
\frac{-3 b_{12}\left(a_{01} b_{21}-a_{10} b_{12}+2 a_{22} b_{00}\right)}{a_{22} b_{21}^{2}}, & k=3 \\
\frac{-3 b_{12}^{2}\left(2 a_{22} b_{00}\left(a_{01} b_{21}-2 a_{10} b_{12}+3 a_{22} b_{00}\right)+\left(a_{01} b_{21}-a_{10} b_{12}\right)^{2}\right)}{a_{22}^{2} b_{21}^{4}}, & k=6\end{cases}
\end{aligned}
$$

Applying Theorem 1 for $q=x^{k}$ we get the sums $s_{k}$ of the $k$-th powers of the $x$-coordinates of the solution points of (4):

$$
\begin{aligned}
& s_{1}=s_{2}=s_{4}=s_{5}=0, \\
& s_{3}=\operatorname{res}_{(3,1)}\left(x^{3}\right)-\operatorname{res}_{(4,3)}\left(x^{3}\right), \\
& s_{6}=\operatorname{res}_{(3,1)}\left(x^{6}\right)-\operatorname{res}_{(4,3)}\left(x^{6}\right) .
\end{aligned}
$$

We now use the Newton formulas

$$
s_{k}-s_{k-1} \sigma_{1}+\cdots+(-1)^{k-1} s_{1} \sigma_{k-1}+(-1)^{k} k \sigma_{k}=0, \quad 1 \leq k \leq 6
$$

to obtain the elementary symmetric polynomials $\sigma_{k}$ : 


$$
\begin{aligned}
& \sigma_{1}=\sigma_{2}=\sigma_{4}=\sigma_{5}=0, \\
& \sigma_{3}=\frac{s_{3}}{3}=\frac{a_{01} b_{21}\left(a_{10} b_{12}+a_{22} b_{00}\right)-\left(a_{10} b_{12}-a_{22} b_{00}\right)^{2}}{a_{10} a_{22} b_{21}^{2}}, \\
& \sigma_{6}=\frac{s_{3}^{2}-3 s_{6}}{18}=\frac{a_{01}^{2} b_{00} b_{12}}{a_{10} a_{22} b_{21}^{2}} .
\end{aligned}
$$

Thus we get a polynomial equation for the $x$ coordinate:

$$
x^{6}-\frac{a_{01} b_{21}\left(a_{10} b_{12}+a_{22} b_{00}\right)-\left(a_{10} b_{12}-a_{22} b_{00}\right)^{2}}{a_{10} a_{22} b_{21}^{2}} x^{3}+\frac{a_{01}^{2} b_{00} b_{12}}{a_{10} a_{22} b_{21}^{2}}=0 .
$$

Since the system (4) is symmetric, an equation for the $y$ coordinate is similar:

$$
y^{6}-\frac{a_{10} b_{12}\left(a_{01} b_{21}+a_{22} b_{00}\right)-\left(a_{01} b_{21}-a_{22} b_{00}\right)^{2}}{a_{01} a_{22} b_{12}^{2}} y^{3}+\frac{a_{10}^{2} b_{00} b_{21}}{a_{01} a_{22} b_{12}^{2}}=0 .
$$

REMARK 2. The fact that the constant terms of the above polynomials are so simple is not a coincidence, but a consequence of a general result due to Khovanskii [Kho99]. Namely, if the Newton polytopes of a system have generic relative position then the product of the $i$-th coordinates of the solution points is always a Laurent monomial in the coefficients of the system (see [Kho99] for details).

REMARK 3. Let us remark that the two equations above can be found by computing the resultants of $f_{1}$ and $f_{2}$ with respect to $x$ and $y$, respectively. However in higher dimensions computing resultants can be a harder problem than finding residues.

\section{References}

[GK02] O. A. Gelfond, A. G. Khovanskii, Toric geometry and Grothendieck residues, Mosc. Math. J., Vol. 2 no. 1, (2002), 99-112.

[GK96] O. A. Gelfond and A. G. Khovanskii, Newton polyhedra and Grothendieck residues, (in Russian) Dokl. Akad. Nauk, 350, no. 3 (1996), 298-300.

[Kho99] A. G. Khovanskii, Newton polyhedra, a new formula for mixed volume, product of roots of a system of equations, Fields Inst. Comm., Vol. 24 (1999), 325-364.

[Sop02] I. Soprounov, Residues and tame symbols on toroidal varieties, math.AG/0203114.

Department of Mathematics and Statistics, University of Massachusetts, Amherst,

MA 01003, USA

E-mail address: isoprou@math.umass.edu 